\title{
Correction: Update of the European consensus on sarcopenia: what has changed in diagnosis and prevalence in peritoneal dialysis?
}

\author{
Maryanne Zilli Canedo Silva • Barbara Perez Vogt • Nayrana Soares Carmo Reis • Jacqueline Costa Teixeira Caramori
}

Published online: 12 December 2019

(C) Springer Nature Limited 2019

\section{Correction to: European Journal of Clinical Nutrition https://doi.org/10.1038/s41430-019-0468-z}

Since publication of the original article the authors noticed two author names were displayed incorrectly. Maryanne Zilli Canedo da Silva should be Maryanne Zilli Canedo Silva, and Nayrana Soares do Carmo Reis should be Nayrana Soares Carmo Reis.

Furthermore, since publication of the original article an erratum regarding the European consensus on sarcopenia was published [1]. This affected the original article's results in several ways. The results affected are listed below.

\section{Abstract}

"The prevalence of sarcopenia ranged from 4 to $10 \%$ according to cut points and references used" should read "The prevalence of sarcopenia remained $4 \%$ according to cut points and references used."

\section{Materials and methods}

Diagnosis of sarcopenia

"Values below $7 \mathrm{~kg} / \mathrm{m}^{2}$ for men and $6 \mathrm{~kg} / \mathrm{m}^{2}$ for women were considered as decreased muscle mass and confirmation of the presence of sarcopenia [3]" should read "Values below $7 \mathrm{~kg} / \mathrm{m}^{2}$ for men and $5.5 \mathrm{~kg} / \mathrm{m}^{2}$ for women were considered as decreased muscle mass and confirmation of the presence of sarcopenia [3]."

\section{Results}

Table 1 was not affected by the change in results and is reproduced here:

"Prevalence of sarcopenia according to the 2019 EWGSOP criteria was $10 \%(n=5)$. Reduced muscle mass alone was prevalent in 20\% $(n=10)$ and reduced muscle function assessed by HGS was frequent in $28 \%(n=14)$ " should read "Prevalence of sarcopenia according to the 2019 EWGSOP criteria was $4 \%(n=2)$. Reduced muscle mass

The original article can be found online at https://doi.org/10.1038/ s41430-019-0468-z. alone was prevalent in $10 \%(n=5)$ and reduced muscle function assessed by HGS was frequent in $28 \%(n=14)$." Figure 1 was affected by the change in results. Both the original Fig. 1 and updated Fig. 1 are produced below:

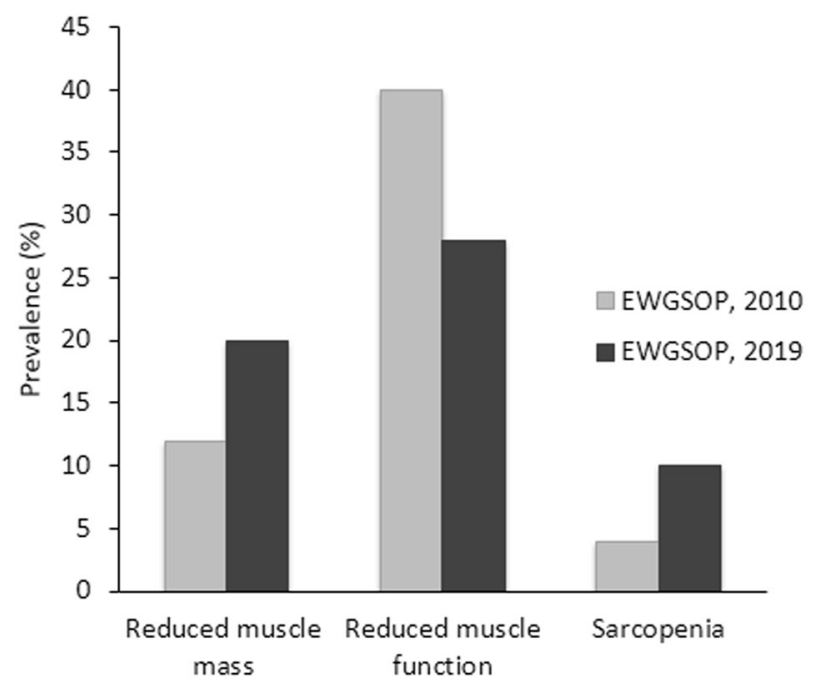

Fig. 1 Prevalence of isolated reduced muscle mass and muscle function, and sarcopenia in PD patients (original).

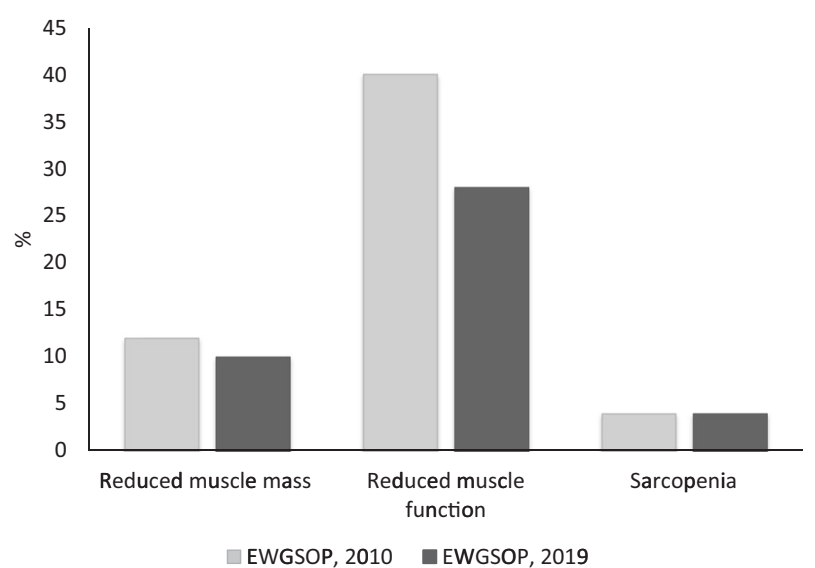

Fig. 1 Prevalence of isolated reduced muscle mass and muscle function, and sarcopenia in PD patients (updated) Discussion 
Table 1 Clinical and nutritional characteristics of the patients

\begin{tabular}{ll}
\hline Variables & $n=50$ \\
\hline Age (years) & $55.74 \pm 16.22$ \\
Gender-Female $[\%(n)]$ & $52(26)$ \\
$\mathrm{Kt} / \mathrm{V}$ & $2.24 \pm 0.48$ \\
Serum Creatinine (mg/dL) & $9.04 \pm 2.79$ \\
Serum Albumin (g/dL) & $3.61 \pm 0.42$ \\
Dialysis vintage (months) & $9.5(5.00-18.00)$ \\
Body weight $(\mathrm{kg})$ & $69.23 \pm 15.63$ \\
Body Mass Index (kg/m²) & $25.99 \pm 4.52$ \\
Handgrip Strength (kg) & $22(18.00-34.25)$ \\
Gait Speed (m/s) & $0.84 \pm 0.23$ \\
Appendicular Skeletal Muscle Mass Index $\left(\mathrm{kg} / \mathrm{m}^{2}\right)$ & $7.21(6.15-8.22)$ \\
Short physical performance battery (points) & $9(8-11)$ \\
\hline
\end{tabular}

"In addition, using the algorithm and cut-off points recommended by EWGSOP consensus update, there was an increase in sarcopenia prevalence in PD patients, which could be underestimated before. The underestimation may increase the risk of worse outcomes in those that hadn't been identified" should read "There is no difference in sarcopenia prevalence according to the 2010 and 2019 criteria. Although consensus updating is important for clinical practice, favoring sarcopenia screening with the measurement of handgrip strength, we found no difference in prevalence with the new criterion."

\section{References}

1. Cruz-Jentoft AJ, Bahat G, Bauer J. et al. Sarcopenia: revised European consensus on definition and diagnosis. Age Ageing. 2019;48:601. https://doi.org/10.1093/ageing/afz046. 